\title{
Análisis de una experiencia de consultoría ético clínica en cuidado intensivo
}

\author{
JUAN PABLO BECA I. ${ }^{1,2}$, ALEJANDRO KOPPMANN A. ${ }^{3}$, PAMELA CHÁVEZ B. ${ }^{3}$, \\ IRIS DELGADO B. ${ }^{2 a}$, SEBASTIÁN SOLAR P. ${ }^{2 b}$
}

\section{Analysis of a clinical ethics consultation experience in intensive care}

Background: Very few patients are presented to ethics committees, and individual ethics consultations are a response to this situation. At the intensive care unit (ICU) in Clinica Alemana, Santiago Chile, an ethics consultation system was organized coordinated with the ethics committee. Aim: To report an evaluation of this ethics consultation system. Material and Methods: Analysis of the first 50 cases analyzed in the consultation system. Analysis of the responses of intensive care physicians to a questionnaire about the main ethical problems that they face in their work. Results: The consultation system is mainly required by the ICU staff, and reports to the ethics committee. Fifty four percent of patients subjected to consultation were aged over 80 years. The main diagnoses were neurological, oncological or cardiopulmonary problems. The ethical problems identified were treatment limitation (62\%), proportionality or futility (42\%), need of a peaceful death (36\%), lack of anticipated decisions (28\%), disagreement between physicians and patient's family (24\%), undefined subrogation (14\%), and abuse of public resources (14\%). Twenty six of 31 ICU physicians answered the questionnaire, using a 1 ( $\min$ ) to 7 (max.) scale. They found that consultation is helpful for decision making (6.3), useful for improving ethical perception (6.0), supportive for staff (6.5), good for patients (6.3), supportive for families (6.7), and timely performed (5.2). Conclusions: As a complement for the ethics committee's work, consultation is a valid alternative for ethics counselling and a support for physicians and patient's families. Its implementation depends on the particularities of each health institution.

(Rev Med Chile 2010; 138: 815-820).

Key words: Ethics Committees, clinical; Ethics, medical; Intensive care units.
'Centro de Bioética.

${ }^{2}$ Facultad de Medicina Clínica Alemana Universidad del Desarrollo. Santiago de Chile. ${ }^{3}$ Clínica Alemana.

${ }^{a}$ Estadístico, Licenciado en Matemáticas, Magister en Bioestadísticas.

${ }^{\mathrm{b}}$ Estudiante de Medicina.

Recibido el 26 de noviembre de 2009, aceptado 6 el julio de 2010

Correspondencia a: Juan Pablo Beca I. Av, Las Condes 12438, Lo Barnechea, Santiago Chile (562) 3279157 E-mail: jpbeca@udd.cl
L os problemas ético-clínicos son cotidianos y la experiencia muestra que los casos que se presentan a los comités de ética son los más extremos o conflictivos y su número es escaso, con un promedio de 3 anuales en Estados Unidos de Norteamérica ${ }^{1}$. En Chile no se ha cuantificado, pero en diferentes instituciones su número varía entre 3 y 20 casos anuales. Entre las razones para el bajo número de casos que se presenta a los comités cabe mencionar: demora entre la solicitud y la posibilidad de reunión del comité, tiempo necesario para preparar y presentar el caso, temor de algunos médicos clínicos a sentirse evaluados o juzgados, desconocimiento de las ventajas de recibir recomendaciones para sus decisiones, y las diferentes visiones o experiencias previas ${ }^{2}$. Esto explica que se formulen frecuentes preguntas informales o "consultas de pasillo" a algún miembro del comité, con lo cual se omite un análisis sistemático y el registro en actas y en la ficha clínica. Como respuesta a esta situación, en algunos países, se han establecido sistemas de consultoría ética individual $^{3}$. Su objetivo es identificar y analizar los problemas éticos de casos particulares, facilitar la resolución de conflictos, disminuir el estrés moral de profesionales o familiares, y educar a través de las recomendaciones que se proponen ${ }^{4}$. La principal ventaja es la eficiencia y rapidez, permitiendo 
incluso organizar sistemas de turno cuando hay varios consultores en una institución. Hay limitaciones evidentes, una de las cuales es depender mucho de las cualidades personales del consultor. Por eso se ha propuesto que los consultores, además de conocimientos de Bioética y de atributos de compasión y tolerancia, deben tener capacidad para comunicarse bien, analizar y documentar los casos, generar consensos y educar a los implica$\mathrm{dos}^{5}$. Otra limitación, frecuentemente referida, es la falta de deliberación multidisciplinaria que caracteriza a los comités de ética asistencial ${ }^{6-8}$. Pero, al igual que los comités, los sistemas de consultoría ético-clínica han sido escasamente evaluados por la complejidad de su labor y por la dificultad de aplicar o adecuar métodos de evaluación ${ }^{1,9-11}$.

Recientemente se implementó en una clínica privada chilena un sistema de consultoría éticoclínica para la unidad de pacientes críticos, mediante una forma de trabajo coordinado con el comité de ética asistencial. En nuestro medio no se han comunicado otros sistemas de consultoría ética. Con el propósito de darlo a conocer se presenta un análisis de esta experiencia, describiendo su sistema de trabajo, casos representativos, los problemas éticos más frecuentes, y la evaluación que de ella hicieron los médicos intensivistas.

\section{Método}

La metodología corresponde a un diseño de tipo descriptivo analítico, un análisis cuantitativo de los problemas más frecuentes y una evaluación realizada por médicos intensivistas.

El universo del estudio fueron los primeros 50 casos de consultoría ético-clínica realizadas en la Unidad de Cuidado Intensivo de Clínica Alemana de Santiago entre abril 2008 y enero 2009. Los diagnósticos principales de los pacientes se agruparon en: cuadros neurológicos, cáncer, patología cardíaca o pulmonar, y un grupo de "otros diagnósticos". Para la descripción de los problemas éticos más frecuentes se sistematizó la identificación independiente de tres de los autores (JPB, AK, PC), cuyas descripciones fueron unificadas en su terminología, promediadas y posteriormente jerarquizadas por frecuencia. El resultado se presenta en porcentajes de ocurrencia. De forma similar se consensuaron tres casos representativos cuyo relato permite comprender mejor las situaciones clínicas en que se solicitó consulta ética.

La evaluación de la consultoría se realizó mediante una encuesta anónima cuyas respuestas se pidieron, previo consentimiento informado, a todos los médicos de la UCI. Después de preguntas para conocer su experiencia, el tiempo de trabajo en esa unidad y si se había realizado consulta ética en pacientes a su cargo, se les pidió evaluar la consultoría, aplicando una escala de 1 a 7 entre total desacuerdo y total acuerdo o satisfacción, sobre 6 aspectos: utilidad para la toma de decisiones, contribución para percibir mejor los aspectos éticos, apoyo a los médicos, beneficio para los pacientes, apoyo a familiares, y acerca de la oportunidad de solicitud de consultas éticas. La encuesta también incluyó dos preguntas de respuesta abierta. El análisis estadístico se realizó calculando estadísticas de tendencia central y de dispersión. Los análisis estadísticos se realizaron con el software SPSS versión 16.0.

Como forma de trabajo de la consultoría individual se nombró un médico consultor, quien coordina su labor con el Comité de Etica. Las consultas éticas fueron solicitadas por el equipo médico tratante o por familiares y asumidas como interconsultas sin cobro a pacientes. Después de conocer los antecedentes clínicos y el contexto de cada caso, el consultor analizó los problemas éticos y escribió su opinión y recomendaciones en la ficha clínica. Todos los casos se registraron en una base de datos con su información básica, diagnósticos, recomendaciones y evolución, categorizándolos según los problemas éticos principales.

\section{Resultados}

En este período se realizaron 50 interconsultas. En 3 de los primeros 15 casos y en 27 de los 35 siguientes se realzaron reuniones con familiares. Se realizó seguimiento y frecuentemente nuevos análisis con médicos y familiares. Periódicamente se informaron los casos al comité de ética y cuatro de ellos, por su mayor complejidad, fueron analizados en sus reuniones.

Del total de casos, más de la mitad tenía 80 o más años de edad (Tabla 1). Si bien todos los enfermos tenían patología múltiple, sus diagnósticos principales, agrupados por frecuencia se detallan en la Figura 1. Se identificaron variados 
Tabla 1. Sexo y edad de los 50 pacientes presentados a consultoría ética en UCI

\begin{tabular}{|lcc|}
\hline & n & \% \\
\hline Hombres & 27 & 54 \\
\hline Mujeres & 23 & 46 \\
\hline Menos de 70 años & 18 & 36 \\
\hline $0-80$ años & 6 & 12 \\
\hline Más de 80 años & 26 & 52 \\
\hline
\end{tabular}

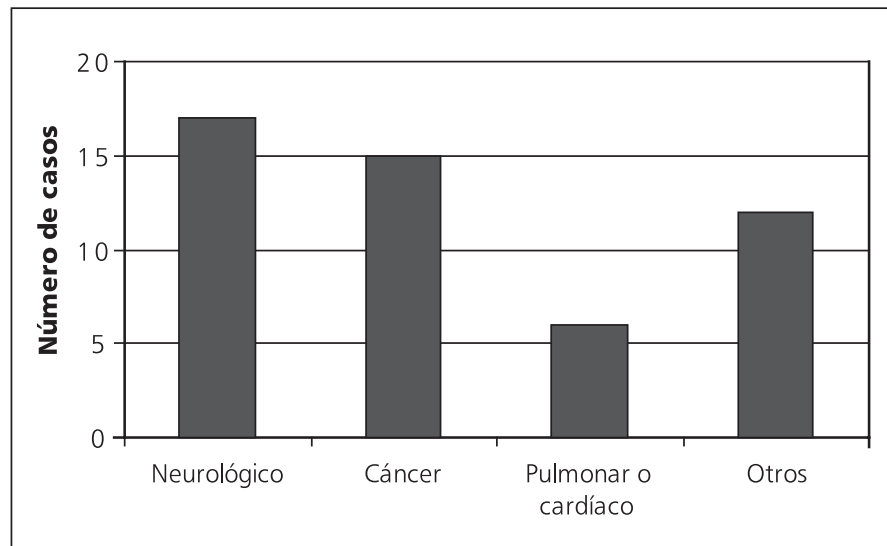

Figura 1. Grupos diagnósticos principales en 50 pacientes de UCl presentados a consultoría ética.

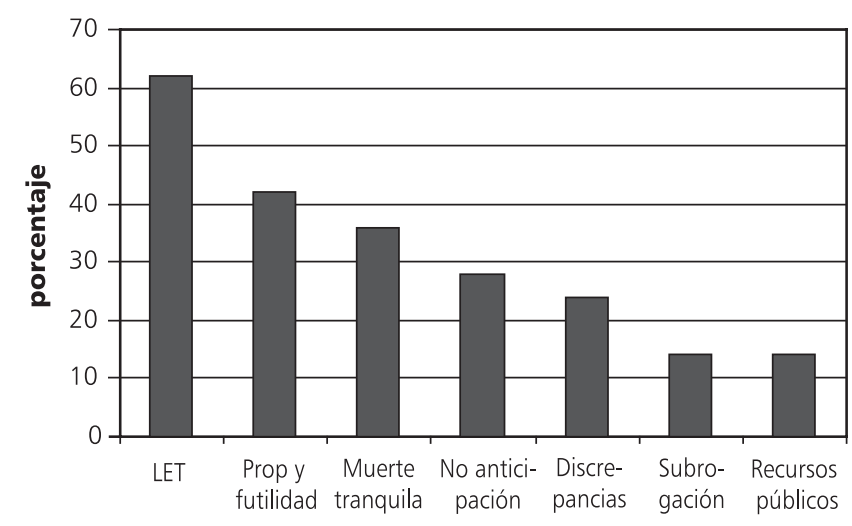

Figura 2. Problemas ético-clínicos más frecuentes en 50 pacientes de UCI presentados a consultoría ética. LET: limitación del esfuerzo terapéutico. y múltiples problemas ético-clínicos, aún cuando generalmente se consultó sólo por uno de ellos. Los más frecuentes fueron los relacionados con limitación del esfuerzo terapéutico y problemas de proporcionalidad o futilidad de tratamiento. Con menor frecuencia el problema principal fue cómo favorecer una muerte tranquila, la falta de anticipación de conductas dificultando limitar tratamientos, o la discrepancia de opiniones entre el equipo tratante y los familiares del enfermo. En otros casos el problema principal fue definir la subrogación del enfermo, o un tema de justicia por el uso desproporcionado de recursos públicos. El porcentaje de ocurrencia se muestra en la Figura 2. Otros problemas éticos, presentes con menor frecuencia, incluyeron inseguridad familiar, ausencia de médico tratante, y rechazo de tratamiento por parte del enfermo.

Para una mejor comprensión del tipo de situaciones clínicas en las cuales se solicitó consultoría ética se describen los tres casos representativos seleccionados:

\section{Caso 1}

Mujer 64 de años, portadora de HTA, cardiopatía coronaria, monorrena quirúrgica, síndrome nefrótico por Nefropatía Membranosa en tratamiento inmunosupresor. Ingresó a UCI en shock séptico secundario a neumonia neumocócica. Evolucionó en forma tórpida, requiriendo apoyo de diálisis por catéter, sufrió infarto agudo al miocardio y sobreinfección micótica sistémica. $\mathrm{Al} 23^{\circ}$ día de hospitalización continuaba con ventilación no invasiva y drogas vasoactivas. Era portadora de enfermedad depresiva grave, con dos hospitalizaciones psiquiátricas, últimamente estable. Era dependiente en algunas actividades básicas, no salía sola, no manejaba sus finanzas ni sus medicamentos. Vivía, con su esposo 
oxígeno dependiente, en casa de una hija. Se solicitó consultoría ética para definir limitación de esfuerzo terapéutico, pues el equipo de intensivos consideraba que un retroceso en su evolución sería un evento final.

\section{Caso 2}

Hombre de 75 años, portador de insuficiencia cardíaca, insuficiencia renal en diálisis peritoneal por tres años, amaurosis por degeneración macular, con antecedentes de cáncer de próstata tratado e infecciones urinarias frecuentes. Ingresó en shock séptico por peritonitis asociada a peritoneodiálisis, requirió diálisis por catéter, drogas vasoactivas, ventilación mecánica y alimentación parenteral. El paciente no presentaba deterioro cognitivo previo a su ingreso, era asistido por cuidadora y la familia lo consideraba bien adaptado a su situación. Vivía con esposa y tenía dos hijos.

La familia solicitó consultoría ética para limitar tratamientos, pues el paciente había expresado, verbalmente y por documento notarial, su deseo de no ser sometido a tratamientos extraordinarios ante una enfermedad grave. Además manifestó, al ingreso, que si se agravaba lo "dejen morir en paz".

\section{Caso 3}

Mujer de 41 años, casada con vida familiar estable y dos hijos adolescentes, que fue encontrada en el baño de su casa en estado de sopor profundo. En UCI se diagnosticó intoxicación por monóxido de carbono. La paciente evolucionó grave con sepsis de foco pulmonar. Requirió apoyo vasoactivo, ventilación mecánica y diálisis. La evaluación neurológica al $15^{\circ}$ día determinó daño cortical severo con evolución probable hacia estado vegetativo. $\mathrm{Su}$ esposo solicitó limitación de esfuerzo terapéutico, incluido el retiro del respirador, pero el equipo médico estaba inseguro.

La encuesta a los médicos fue respondida por 26 de los 31 médicos de la unidad de pacientes críticos $(83,8 \%)$. En promedio tenían 17 años de profesión con 12 años trabajando en cuidado intensivo. Tres de ellos no habían tenido a su cargo pacientes en quienes se realizó consultoría, por lo que sus respuestas se excluyeron del análisis. La calificación que realizaron los médicos, en escala 1 a 7, demostró que la consultoría fue considerada útil para la toma de decisiones complejas (promedio 6,3$)$, un apoyo a los médicos $(6,5)$, mejora la capacidad de percepción de aspectos éticos $(6,0)$,
Tabla 2. Evaluación de consultoría realizada por médicos de $\mathrm{UCl}$ intensivistas aplicando escala 1 a 7

\begin{tabular}{|lcc|}
\hline $\begin{array}{l}\text { Ayuda de la } \\
\text { consultoría para }\end{array}$ & Promedio & $\begin{array}{c}\text { Desviación } \\
\text { Estándar }\end{array}$ \\
$\begin{array}{l}\text { Utilidad para decisio- } \\
\text { nes complejas }\end{array}$ & 6,3 & 0,6 \\
$\begin{array}{l}\text { Grado de apoyo a } \\
\text { médicos }\end{array}$ & 6,5 & 0,7 \\
\hline $\begin{array}{l}\text { Mejorar capacidad de } \\
\text { percepción }\end{array}$ & 6,0 & 1,1 \\
$\begin{array}{l}\text { Beneficio para el } \\
\text { enfermo }\end{array}$ & 6,3 & 1,0 \\
\hline $\begin{array}{l}\text { Apoyo para la familia } \\
\text { Solicitud oportuna }\end{array}$ & 6,7 & 0,5 \\
\hline
\end{tabular}

beneficia al enfermo $(6,3)$, apoya a la familia $(6,7)$, y que fue solicitada oportunamente $(5,2)$. Tabla 2.

En sus respuestas abiertas los médicos sugirieron que las consultas éticas se soliciten con mayor anticipación, que se incorpore a los médicos tratantes externos y que se realicen visitas ético-clínicas regulares. Además propusieron que se les capacite en bioética y que la consultoría se extienda a servicios como oncología, neurología y pediatría.

\section{Discusión}

Los médicos clínicos suelen considerar a los comités de ética como instancias distantes de su práctica o como una interferencia en su labor, razón por la cual prefieren las consultas éticas informales o las consultorías individuales ${ }^{11,12}$. Los problemas éticos son frecuentes en pacientes críticos o terminales pero a los comités de ética sólo se presentan casos especialmente complejos que generan conflictos éticos. Esta experiencia confirma lo anterior pues a este comité de ética sólo se presentan entre 3 y 5 casos anuales. Los casos representativos presentados son casos habituales que probablemente no habrían sido presentados al comité. Similar es la experiencia reportada por otros autores, apoyando la justificación de consultorías individuales como forma de ayuda para decisiones ético-clínicas que incluyen no sólo situaciones de conflicto, sino también casos 
en los cuales existe necesidad de reasegurar una determinación médica o familiar ${ }^{2,8}$. Esta experiencia mostró que el objetivo inicial de apoyo a los profesionales en sus decisiones se amplió al apoyo a pacientes o familiares, coincidiendo con otros estudios ${ }^{13}$.

El análisis de los comités de ética constituye una deliberación plural y multidisciplinaria que caracteriza su método. La dificultad es que su labor es inevitablemente lenta, más distante de los clínicos y consecuentemente analizan muy pocos $\operatorname{casos}^{1}$. Las consultorías éticas en cambio son más cercanas, asumen mayor número de casos y pueden conocer más su contexto clínico y social, pero carecen del análisis multidisciplinario ${ }^{6,7,14}$. El método de análisis de la consultoría individual es eminentemente práctico e integra elementos de bioética de principios, casuística y ética del cuidado, en base a un razonamiento moral particularmente sensible al contexto ${ }^{8,15,16}$. La deliberación se realiza con los clínicos y las familias, para lo cual el consultor necesita actuar con máxima imparcialidad evitando actuar como un "experto en ética" quien recomienda desde una sola perspectiva. Las cualidades y competencias personales del consultor tienen, por lo tanto, máxima relevancia ${ }^{5}$. La alternativa entonces no debería ser "comités o consultores" sino un modelo de "comités y consultores", trabajando de manera complementaria, apoyando y estimulando a los clínicos a reconocer problemas bioéticos y seleccionando los casos a ser analizados por los comités. Es importante insistir en que el responsable de abordar y resolver los problemas éticos es el staff clínico, decidiendo de manera compartida con pacientes o familiares, con la ayuda de comités y de consultores.

Si bien los sistemas de consultoría ética necesitan ser evaluados, no hay propuestas metodológicas uniformes para hacerlo ${ }^{2,11,17}$. Por esta razón la mayoría de las comunicaciones son descriptivas y observacionales, seguidas de discusiones analíticas ${ }^{1,18-21}$. Un primer resultado positivo de este estudio es el aumento del número de casos consultados. También lo es el hecho que la consultoría sigue un método de trabajo complementario con el comité de ética. El tipo de problemas o dudas éticas por las cuales se solicitaron consultorías mostró, como en otras series, que los problemas más frecuentes son dudas concretas relacionadas con limitación y proporcionalidad, así como con decisiones de tratamiento al final de la vida. El análisis más amplio y el mayor conocimiento del contexto de cada caso permiten abordar otros aspectos y proponer las respectivas conductas de apoyo. Los temas de justicia, si bien no fueron motivo inicial de consulta, constituyen un problema no comunicado en otras publicaciones. La encuesta de opinión de los médicos, respondida por un alto porcentaje de ellos, mostró un alto grado de satisfacción y destaca que la consultoría fue considerada útil para las decisiones y un apoyo a médicos y a familiares de pacientes. La oportunidad de su solicitud fue considerada como algo tardía, lo cual se suma a las opiniones abiertas que sugieren que los consultores participen en las visitas clínicas, lo cual acercaría aún más la bioética a la práctica clínica, junto con aumentar el reconocimiento de aspectos éticos o dudas presentes en muchos casos.

La evaluación más compleja es la percepción de los familiares. En este estudio no fue posible diseñar una metodología para este efecto, aun cuando se recibieron numerosas opiniones espontáneas muy favorables. Estudios que han realizado evaluaciones de familiares han confirmado resultados positivos, pero también han encontrado ocasionales opiniones desfavorables cuyo análisis muestra que están influidos por factores de frustración por la muerte $\mathrm{u}$ otros resultados negativos del tratamiento ${ }^{22,23}$. La necesidad de conocer las opiniones de familiares en nuestro medio está abierta, definiendo una metodología que respete la sensibilidad de cada caso.

Finalmente, y a modo de conclusión, puede afirmarse que la consultoría ético-clínica individual, o la realizada por dos o tres consultores, constituyen una forma válida y factible de asesoría en las decisiones clínicas y un apoyo a médicos y familiares. Cumple también un rol de sensibilización y formación para los profesionales que trabajan en UCI u otros servicios a los que se extienda. La consultoría ética se entiende como un complemento de los comités de ética asistencial que aportan una deliberación ética multidisciplinaria de los casos más complejos y un rol de análisis y propuesta normativa para situaciones institucionales específicas. Cabe insistir en que un sistema de consultoría ética individual depende de las condiciones particulares de cada institución y está ligado a las cualidades personales de cada consultor, de manera que no es posible formular recomendaciones generalizables. 


\section{Referencias}

1. Fox E, Myers S, Pearlman RA. Ethics consultation in United States hospitals: a national survey. Am J Bioeth 2007; 7: 13-25.

2. Aulisio MP, Moore J, Blanchard M, Bailey M, Smith D. Clinical ethics consultation and ethics integration in an urban public hospital. Camb Q Healthc Ethics 2009; 18: 371-83.

3. Pfafflin M, Kobert K, Reiter-Theil S. Evaluating Clinical Ethics Consultation: a European Perspective Camb Q Healthc Ethics 2009; 18: 408-19.

4. Ethics Committees and Ethics Consultation. Ethics in Medicine. University of Washington School of Medicine. Disponible en: http://depts.washington.edu/bioethx/ topics/ethics.html

5. Aulisio MP, Arnold RM, Youngner SJ. Health care ethics consultation: nature, goals, and competencies. A position paper from the Society for Health and Human Values-Society for Bioethics Consultation Task Force on Standards for Bioethics Consultation. Ann Intern Med 2000; 133: 59-69.

6. Beca JP. Comités de Ética o Consultores de ética clínica. Bioètica \& Debat 2008; 54: 1-5.

7. Couceiro A. Comités de ética o consultores de ética: ¿qué es lo mejor para las instituciones sanitarias?. Bioètica \& Debat 2008; 54: 10-5.

8. Richter G. Clinical Ethics as Liaison Service: Concepts and Experiences in Collaboration with Operative Medicine. Camb Q Healthc Ethics 2009; 18: 360-70.

9. Hurst SA, Hull SC, DuVal G, Danis M. How physicians face ethical difficulties: A qualitative analysis. J Med Ethics 2005; 31: 7-1.

10. Schneiderman LJ, Gilmer T, Teetzel HD. Impact of Ethics Consultations in the Intensive Care Setting: A Randomized, Controlled Trial. Crit Care Med 2000; 28 : 3920-3924ics 2008; 19: 204-13.

11. Agich GJ, Reither-Thiel S. Guest Editorial: Encouraging the Dialogue. Camb Q Healthc Ethics 2009; 18: 333-7.

12. Slowter A. Ethics Case Consultation in Primary Care: Contextual Challenges for Clinical Ethicists. Camb Q Healthc Ethics 2009; 18: 397-405.

13. Agich GJ. Why Quality Is Addressed So Rarely in Clinical Ethics Consultation. Camb Q Healthc Ethics 2009; 18: 339-46.

14. Bioética\&Debat. ¿Con quién asesorarse sobre los problemas éticos?. Editorial. Bioética\&Debat 2008; 54: 2.

15. Agich GJ. The question of method in ethics consultation. Am J Bioeth 2001; 1: 31-41.

16. Dudzinski DM. The practice of a clinical ethics consultant. Public Aff Q 2003; 17: 121-39.

17. Chen Y-Y CY-C. Evaluating ethics consultation: randomized controlled trial is not the right tool. J Med Ethics 2008; 34: 594-7.

18. Childers JW, Demme R, Greenlaw J, King DB, Quill T. A Qualitative Report of Dual Palliative Care/Ethics Consultations: Intersecting Dilemmas and Paradigmnatic Cases. J Clin Ethics 2008; 19: 204-13.

19. DuVal G, Clarridge B, Gensler G, Danis M. A national survey of U.S. internists' experiences with ethical dilemmas and ethics consultation. J Gen Intern Med 2004; 19 : 251-8.

20. Schneiderman LJ, Gilmer T, Teetzel HD, Dugan DO, Blusyein J, Cranford R, Briggs KB. Effect of ethics consultations on nonbeneficial life-sustaining treatments in the intensive care setting: a randomized controlled trial. JAMA 2003; 290: 1166-72.

21. Swetz KM, Crowley ME, Hook C, Mueller PS. Report of 255 clinical ethics consultations and review of the literature. Mayo Clin Proc 2007; 82: 686-91.

22. Schneiderman LJ, Gilmer T, Teetzel HD, Dugan DO, Goodman-Crews P, Cohn F. Dissatisfaction with ethics consultations: The Anna Karenina principle. Camb Q Healthc Ethics 2006: 15: 101-6.

23. Howe EG. Beyond the State of the Art in Ethics Consultation. The Journal of Clinical Ethics 2009; 20: 203-11. 